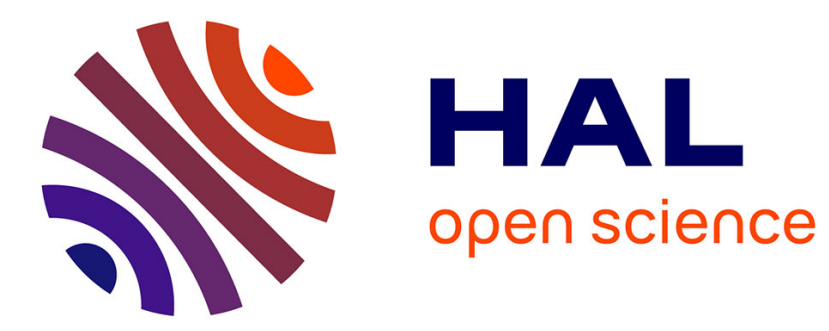

\title{
Computational nonlinear thermomechanical model of large light partition walls based on experimental analyzes and probabilistic models
}

T. T. Do, Christian Soize, J.-V. Heck

\section{- To cite this version:}

T. T. Do, Christian Soize, J.-V. Heck. Computational nonlinear thermomechanical model of large light partition walls based on experimental analyzes and probabilistic models. Computer-Aided Civil and Infrastructure Engineering, 2013, 28 (2), pp.81-97. 10.1111/j.1467-8667.2012.00782.x · hal-00743717

\section{HAL Id: hal-00743717 \\ https://hal.science/hal-00743717}

Submitted on 19 Oct 2012

HAL is a multi-disciplinary open access archive for the deposit and dissemination of scientific research documents, whether they are published or not. The documents may come from teaching and research institutions in France or abroad, or from public or private research centers.
L'archive ouverte pluridisciplinaire HAL, est destinée au dépôt et à la diffusion de documents scientifiques de niveau recherche, publiés ou non, émanant des établissements d'enseignement et de recherche français ou étrangers, des laboratoires publics ou privés. 


\title{
Computational nonlinear thermomechanical model of large light partition walls based on experimental analyzes and probabilistic models
}

\author{
T.T. Do ${ }^{\mathrm{a}, \mathrm{b}}$, C. Soize ${ }^{*, a}$, J.-V. Heck ${ }^{\mathrm{b}}$ \\ ${ }^{a}$ Université Paris-Est, Laboratoire Modélisation et Simulation Multi-Echelle, MSME UMR 8208 CNRS, 5 bd Descartes, \\ 77454 Marne-la-Vallée Cedex 2, France \\ ${ }^{b}$ Université Paris-Est, Centre Scientifique et Technique du Bâtiment, 84 Avenue Jean-Jaures, 77447 \\ Champs-sur-Marne,France
}

\begin{abstract}
This paper is devoted to the construction of a computational nonlinear thermomechanical model of large light partition walls made up of cardboard-plasterboard-cardboard (CPC) plates screwed to a metallic frame, and is submitted to mechanical and thermal loadings induced by fire. The computational model, based on experimental analyzes and a probabilistic modeling, is developed for the prediction of a large light partition wall for (1) its linear thermal behavior, (2) its nonlinear mechanical behavior and (3) its nonlinear thermomechanical behavior.
\end{abstract}

Key words: Screw attachment, plasterboard, cardboard, experimental identification, thermomechanical, fire load

\section{INTRODUCTION}

The simulation of the fire resistance of structures is a great challenge and numerous papers have been published in this field since two decades (see for instance, Kodur et al. (2008), Jones and Wang (2008), Wang (2008)). For several decades, a considerable effort has been carried out to develop the materials which have a good fire resistance. One of the materials construction with the best fire resistance is the multilayer Cardboard-Plasterboard-Cardboard (CPC) plate. The plaster has the feature to undergo two chemical reactions of dehydration during its rise of temperature. Both reactions slow down the rise of temperature of CPC plates. This paper is concerned by large light partition walls made up of CPC plates screwed to metallic frames.

A lot of works have been published concerning the chemical, mechanical, thermal and thermomechanical properties of the CPC plates. For the chemical properties of plaster, the reader is referred to Andersson and Jacksson (1995), Gerlich (1995), Axenenko and Thorpe (1996), Sultan (2003), for the mechanical properties of the plaster and of the CPC plates, to Benouis (1995), Cramer et al. (2003). Concerning the thermal properties of the plaster and of the CPC

\footnotetext{
${ }^{*}$ Corresponding author

Email address: christian.soize@univ-paris-est.fr (C. Soize)
} 
plates, the reader is referred to NIST (1980), Harmathy (1988), Fuller et al. (1992), Mehaffey and Cuerrier (1994), Sultan (1996) for mass density, to Harmathy (1988), Fuller et al. (1992), Mehaffey and Cuerrier (1994), Andersson and Jacksson (1995), Hadjisophocleous (1996), Sul$\tan$ (1996), Konig and Walleij (2000), Thomas (2002) for specific heat, to Harmathy (1988), Mehaffey and Cuerrier (1994), Goncalves et al.(1996), Hadjisophocleous (1996), Sultan (1996), Cooper (1997), Thomas (2002) for thermal conductivity. The thermomechanical properties of the constituents of the CPC plates can be found in Sakji (2006). It should be noted that no experimental data have been published in the open literature concerning mechanical behavior of the screw attachments of the plasterboards for the low and the high temperatures in presence of mechanical forces. In addition, experimental data are missing in the literature concerning the thermal dilatation and the thermal curvature for the CPC plates, for the stress-strain curve in compression and in tensile for low and high temperature of the CPC plates. This is the reason why experiments have been carried out for these missing data in order to complete the database and are represented in this paper. It should also be noted that previous works have been carried out in this field for thermomechanical analysis of plasterboard plates submitted to fire loads and to mechanical forces in deterministic and probabilistic frameworks Sakji (2006), Sakji et al. (2008, 2009). Concerning the screw attachment of CPC plates under a thermal loading, no results concerning modeling and experiments have been proposed in the literature although works have been published for other types of attachments with other materials (see for instance Lackey et al.(2003), Cachim and Franssen (2009).

This paper is devoted to the construction of a computational nonlinear thermomechanical model of a large light partition wall, made up of CPC plates screwed to a metallic frame, at room temperature and at high temperatures, and by its experimental validation. The prime objective of this paper is to develop a computational nonlinear thermomechanical model based on experimental analyzes and a probabilistic modeling to take into account the experimental variability induced by the complexity of such thermomechanical systems. The experimental analyzes allow us to identify (1) the shear behavior of the screw attachment and (2) the thermal, the mechanical and the thermomechanical properties of the CPC plates at room temperature and at high temperatures. The computational model proposed and experimentally identified allows us to analyze the nonlinear thermomechanical behavior of light partitions wall subjected to fire loads in a robust framework with respect to the modeling errors and to the variability of the real thermomechanical system which induces a large variability in experimental data due to the type of materials and the manufacturing processes. In addition, since the proposed semi-physical model for the screw attachments corresponds to an approximation, uncertainties must be taken into account in the model (see for instance PateCornell (1996), Soize (2000), Schueller (2005a, b). For that, the usual parametric probabilistic approach is used (see for instance Beck and Katafygiotis (1998), Beck et al. (2010), Cheung and Beck (2010), Schueller (2005a, b), Soize (2000, 2012), Irfanoglu (2012). The prior probability distributions are then constructed using the Maximum Entropy (MaxEnt) principle (see for instance Jaynes (1957a, b), Kapur and Kesavan (1992), Soize (2010)) of Information Theory (Shannon, 1948; Cover and Thomas, 2006) under the constraints defined by the available information. The identification of the unknown parameters of the prior probability distributions is performed using usual statistical methods (Serfling, 1980; Walter and Pronzato, 1997; Spall, 2003) with the experimental data which leads us to optimal prior probability distributions. The use of the optimal prior probability model for the screw attachments, allows a stochastic model to be developed for the prediction of the nonlinear thermomechanical behavior of a large light partition wall submitted to fire loads and under mechanical forces. 
It should be noted that a simplified model has been introduced and experimentally identified for the following reasons. The introduction of a full computational model of the structure with the screw attachments would require introducing a thermomechanical nonlinear micro-macro model to describe the behavior of the screw between the plasterboard plates and the metallic frame. Such a model would be very difficult to develop and a lot of data would be missing to perform efficient calculations. This is why we did not try to develop such a multiscale approach and we have preferred to construct a computational model at macroscale which includes a semi-physical modeling of the shear behavior of the screws in the plasterboard plates using an experimental analysis and a probabilistic modeling.

The objective of such a research is to validate a computational model in order to replace the experimental certification of the large light partition walls subjected to fire loads by a certification with a computational model. It should be noted that this paper present completely new results for this difficult nonlinear thermomechanical problem related to a very complex mechanical system, the complexity being due to the nature of the materials such has plaster and cardboard, and also due to the type of attachments which are realized with screws. This is the first time that such a computational model, constructed and validated with dedicated experiments, is proposed in the open literature.

\section{EXPERIMENTAL ANALYSIS AND PROBABILISTIC MODELING OF THE SHEAR BEHAVIOR FOR THE SCREW ATTACHMENT}

The experiments have been carried with two types of screw attachment related to a partition wall made up of CPC plates with a single skin or to a partition wall made up of CPC plates with a double skin. The experiments have been done for these two types and can be found in Do (2011). In this paper, for reasons of length, the presentation of results is restricted to the first type. It should be noted (see Do (2011)) that the results obtained for a double skin is similar to the single skin for which the time scale of the thermal loading must be divided by 2 . The experimental analysis and the probabilistic modeling of the shear behavior for the screw attachment which are presented are valid for a given screw and for a given CPC plate. If the screw or the CPC plate are significantly modified, the validity of the results presented should be done using additional measurements. Nevertheless, the choices of the screw and of the CPC plate correspond to normalized technologies and are representative of screws and CPC plates which are commonly used.

\subsection{Experimental results and their analyzes}

For the tests with a thermal loading at high temperature, a test bench has been developed (Sakji, 2006) at Centre Scientifique et Technique du Bâtiment (CSTB). This Thermal Load Bench (TLB) can reproduce a heat flux equivalent to that induced by the thermal loading defined by ISO 834 curve. The temperature is given by

$$
\theta(t)=20+345 \log (8 t+1)
$$

in which, $\theta(t)$ is the difference between the temperature (in Celsius degrees) at time $t$ and the initial temperature, and where $t$ is time (in minutes) for which the measurement is done. Figure 1 


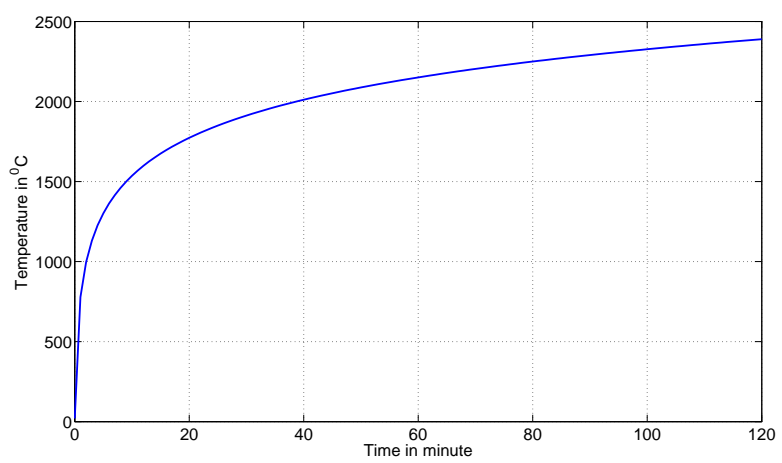

Figure 1: ISO 834 curve: temperature in Celsius degrees as a function of time in minute.

shows the ISO 834 curve.

At room temperature, the experiments have been carried out with 8 samples. The relative displacement $x$ at the screw level, induced by the applied load $y$, is limited to $x_{\max }=0.007 \mathrm{~m}$ which corresponds to the upper limit for which the averaging of the 8 experimental load-displacement curves is monotonic increasing. This limit corresponds to the upper value for the practical applications. It should be noted that each experimental configuration is destroyed after the experiment because the load is applied until the rupture. Consequently, a given experimental configuration cannot be reused for another test and therefore, the experimental error cannot be evaluated and thus, cannot be separated from the variability induced by the samples. Nevertheless, other considerations show that the experimental errors are negligible with respect to the variability for the mechanical system. The experimental results for the 8 samples are represented by 8 loaddisplacement curves (8 thin lines) displayed in Figure 3.

At high temperature, the experiments are carried out in two steps: (1) a thermal loading test is done using the TLB and (2) a mechanical loading test is done using the mechanical setup. At a given time, the first step is stopped and then, the heated sample is mechanically tested. The experiments at high temperature are carried out at several moments which are 900, 1200 and $1500 \mathrm{~s}$. The experimental results of these tests are presented in Figures 4, 5 and 6. For the same value of the displacement, the corresponding values of the load are dispersed and conversely.

\subsection{Nominal model of the shear behavior for the screw attachment and its experimental identification}

The nominal model of the shear behavior is constructed as an algebraic function for which the unknown parameters are identified with the experimental averaging curve. The nominal model of the relative displacement by $x$ induced by the applied load by $y$ is written as

$$
y(x)=a\left\{(x+b)^{\alpha}-b^{\alpha}\right\} .
$$

In Equation (2), $a, b$ and $\alpha$ are three positive real parameters. We introduce the vector $\mathbf{r}=(a, b, \alpha)$ which belongs to an admissible subset $\mathfrak{R}$ of $\mathbb{R}^{3}$ (three-dimensional Euclidean space). Parameter $\mathbf{r}$ is identified using the experimental averaging curve. Since the positive-valued function $x \mapsto y(x)$ 
defined on $\left[0, x_{\max }\right]$ must be strictly concave and since the relative displacement $x$ must be zero if the load applied $y$ is zero, then it can be deduced that, for all $\mathbf{r}$ in $\mathfrak{R}$ and for all $x$ in $\left[0, x_{\max }\right]$, one has

$$
\left\{\begin{array}{l}
y(x) \geq 0 \\
y(0)=0 \\
y^{\prime}(x)=\alpha a(x+b)^{\alpha-1}>0 \\
y^{\prime \prime}(x)=\alpha(\alpha-1) a(x+b)^{\alpha-2}<0
\end{array}\right.
$$

in which $y^{\prime}$ and $y^{\prime \prime}$ are the first and the second derivative of function $y$ with respect to $x$. From Equation (3), it can easily be deduced that parameters $a, b$ and $\alpha$ have to be such that

$$
a>0, \quad b>0, \quad 0<\alpha<1,
$$

which shows that $\mathfrak{R}=] 0,+\infty[\times] 0,+\infty[\times] 0,1[$. The nominal model is identified with the experimental averaging curve using the least-square method,

$$
\mathbf{r}_{0}=\arg \min _{\mathbf{r} \in \mathfrak{R}} \int_{0}^{x_{\max }}\left(y(x ; \mathbf{r})-\underline{y}^{\exp }(x)\right)^{2} d x,
$$

in which $\mathbf{r}_{0}=\left(a_{0}, b_{0}, \alpha_{0}\right)$ and where, for $x$ in $\left[0, x_{\max }\right]$,

$$
\underline{y}^{\exp }(x)=\left(1 / v_{\text {exp }}\right) \sum_{\ell=1}^{v_{\text {exp }}} y^{\text {exp }, \ell}(x)
$$

is the experimental averaging curve constructed with the experimental curves $y^{\exp , 1}(x), \ldots, y^{\exp , v_{\exp }}(x)$. The nominal model is then defined by

$$
y_{0}(x)=a_{0}\left\{\left(x_{0}+b_{0}\right)^{\alpha_{0}}-b_{0}^{\alpha_{0}}\right\} .
$$

For room temperature, the optimal value of parameter $\mathbf{r}_{0}$ is $\left(a_{0}, b_{0}, \alpha_{0}\right)=(32108,0.01905,0.00294)$. The comparison between the nominal model $y_{0}(x)$ and the experimental averaging curve $\underline{y}^{\exp }(x)$ is presented in Figure 2.

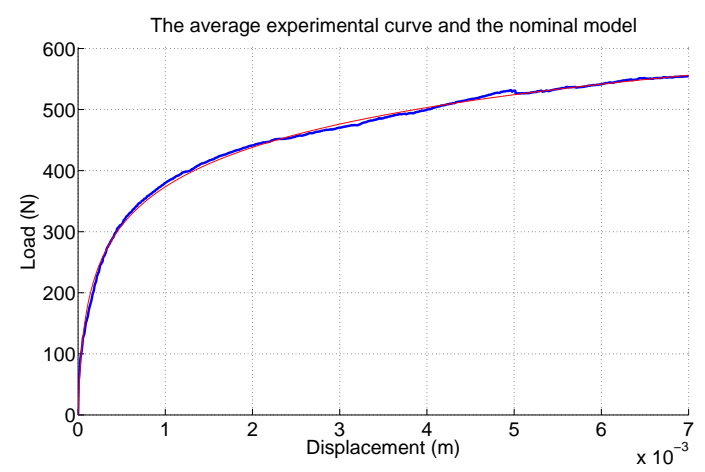

Figure 2: Comparison of the experimental averaging curve $y^{\exp }(x)$ (thick line) with the nominal model $y_{0}(x)($ thin line). 


\subsection{Construction of the prior probability model to take into account variability and un- certainties}

As explained in Section 1, the variability of the experimental data and the model uncertainties are taken into account in modeling the parameters $a$ and $b$ by two independent random variables $A$ and $B$ for which the mean values are $E\{A\}=a$ and $E\{B\}=b$, where $E$ is the mathematical expectation. As explained in Section 1, the prior probability distributions of random variables $A$ and $B$ are constructed using the Maximum Entropy (MaxEnt) principle of Information Theory under the constraints defined by the available information. Since there is no available information concerning the statistical dependence of $A$ and $B$, using the MaxEnt principle, it is proven that the best probabilistic model which maximizes the uncertainties correspond to the one for which the random variables $A$ and $B$ are statistically independent (Kapur and Kesavan, 1992; Soize, 2000). Parameter $\alpha$ is not modeled by a random variable because $\alpha$ is related to the curvature of the random curve which is robust with respect to the variabilities. This deterministic parameter will be identified using the experimental data (see Section 2.4). The deterministic Equation (2) is replaced by the random equation,

$$
Y(x)=A\left\{(x+B)^{\alpha}-B^{\alpha}\right\} .
$$

It should be noted that $E\{Y(x)\}$ is different from $\underline{a}\left[(x+\underline{b})^{\alpha}-\underline{b}^{\alpha}\right]$. For physical reason, $Y$ must be a second-order random variable that is to say, must be such that $E\left\{Y^{2}\right\}<+\infty$. It can be verified that this condition is satisfied if $E\left\{A^{2}\right\}<+\infty$ and if $E\left\{B^{2}\right\}<+\infty$. From Equation (8), it can be deduced that, if the applied load $y$ is given, then the relative displacement $x$ becomes a random variable $X$ such that

$$
X=\left(\frac{y}{A}+B^{\alpha}\right)^{1 / \alpha}-B
$$

Similarly, for physical reasons, $X^{\alpha}$ must be a second-order random variable for all $\alpha$ in ]0,1[ which means that $E\left\{X^{2 \alpha}\right\}<+\infty$. Such a condition is satisfied if $E\left\{A^{-2}\right\}<+\infty$. In addition, this last condition implies that $E\left\{A^{2}\right\}<+\infty$.

Prior probability distribution of random variable $A$. For the random variable $A$, the available information is then defined as follows: (i) the support of the probability distribution of $A$ is ] $0,+\infty$ [, (ii) the mean value of $A$ is $E\{A\}=a$ and (iii) the random variable $A$ must be such that $E\left\{A^{-2}\right\}<+\infty$. The use of the MaxEnt principle under these three constraints yields the probability density function $p_{A}$ of $A$ which is written as

$$
\begin{aligned}
p_{A}\left(a ; \underline{a}, \delta_{A}\right)=\mathbf{1}_{] 0,+\infty[}(a) \frac{1}{\underline{a}}\left(\frac{1}{\delta_{A}^{2}}\right)^{1 / \delta_{A}^{2}} \\
\times \frac{1}{\Gamma\left(1 / \delta_{A}^{2}\right)}\left(\frac{a}{a}\right)^{1 / \delta_{A}^{2}-1} \exp \left(-\frac{a}{\underline{a} \delta_{A}^{2}}\right),
\end{aligned}
$$

in which $\delta_{A}=\sigma_{A} / \underline{a}<1 / \sqrt{2}$ is the coefficient of variation of $A$, where $\sigma_{A}$ is the standard deviation of $A$, where $\Gamma$ is the Gamma function and where $\mathbf{1}_{K}(a)=1$ if $a \in K$ and $=0$ if $a \notin K$.

Prior probability distribution of random variable $B$. For the random variable $B$, the available information is: (i) the support of the probability distribution of $B$ is ]0, $+\infty$ [, (ii) the mean value of $B$ is $E\{B\}=\underline{b}$ and (iii) the random variable $B$ must be such that $E\left\{B^{2}\right\}=\underline{b}^{2}\left(1+\delta_{B}^{2}\right)<+\infty$. The 
use of the MaxEnt principle under these three constraints yields the probability density function $p_{B}$ of $B$ which can be written as,

$$
p_{B}\left(b ; \underline{b}, \delta_{B}\right)=\mathbf{1}_{] 0,+\infty[}(b) C_{0} \exp \left(-\lambda_{1} b-\lambda_{2} b^{2}\right),
$$

where $\left(C_{0}, \lambda_{1}, \lambda_{2}\right)$ are given in solving the following system of nonlinear algebraic equations,

$$
\begin{aligned}
& C_{0} \int_{0}^{+\infty} b \exp \left(-\lambda_{1} b-\lambda_{2} b^{2}\right) d b=\underline{b}, \\
& C_{0} \int_{0}^{+\infty} b^{2} \exp \left(-\lambda_{1} b-\lambda_{2} b^{2}\right) d b=\underline{b}^{2}\left(1+\delta_{B}^{2}\right), \\
& C_{0} \int_{0}^{+\infty} \exp \left(-\lambda_{1} b-\lambda_{2} b^{2}\right) d b=1 .
\end{aligned}
$$

Parameters of the prior probability distributions. The prior probability density functions $p_{A}$ and $p_{B}$ depend only on the parameters

$$
\underline{\mathbf{r}}=(\underline{a}, \underline{b}, \alpha), \boldsymbol{\delta}=\left(\delta_{A}, \delta_{B}\right),
$$

for which parameter $\underline{\mathbf{r}}$ belongs to the admissible set $\mathfrak{R}$ and parameter $\delta$ belongs to an admissible set denoted by $\Delta$. The dispersion parameter $\delta$ allows the dispersion induced by variability and uncertainties to be controlled. These parameters are unknown and will be identified in Section 2.4 using experimental data.

\subsection{Experimental identification}

The parameters $\underline{\mathbf{r}}$ and $\boldsymbol{\delta}$ (introduced in Section 2.3) are identified using the experimental curves $y^{\exp , 1}(x), \ldots, y^{\exp , v_{\exp }}(x)$ for $x$ in $\left[0, x_{\max }\right]$. The methodology used for the identification is carried out in two steps. The first one is the calculation of $\mathbf{r}_{0}$ with Equation (5). This optimal value is used as an initial value for the second step. The second step consists in simultaneously identifying $\underline{\mathbf{r}}$ and $\boldsymbol{\delta}$ with the maximum likelihood method. This non convex optimization problem is solved by using the trial method around the initial values $\underline{\mathbf{r}}=\mathbf{r}_{0}$ and $\boldsymbol{\delta}=\boldsymbol{\delta}_{0}$ with $\boldsymbol{\delta}_{0}$ an adapted initial value. Let

$$
J(\underline{\mathbf{r}}, \boldsymbol{\delta})=\int_{0}^{x_{\max }}\left(Y(x ; \underline{\mathbf{r}}, \boldsymbol{\delta})-\underline{y}^{\exp }(x)\right)^{2} d x
$$

be the random variable whose probability density function is denoted by $\eta \mapsto p_{J}(\eta ; \underline{\mathbf{r}}, \boldsymbol{\delta})$ and where $y^{\exp }(x)$ is defined by Equation (6). For $\ell=1, \ldots, v_{\exp }$, let $J_{\ell}^{\exp }$ be the corresponding experimental data such that

$$
J_{\ell}^{\exp }=\int_{0}^{x_{\max }}\left(y^{\exp , \ell}(x)-\underline{y}^{\exp }(x)\right)^{2} d x .
$$

Let $\mathcal{L}(\underline{\mathbf{r}}, \boldsymbol{\delta})$ be the log-likelihood function defined on $\mathfrak{R} \times \Delta$ such that

$$
\mathcal{L}(\underline{\mathbf{r}}, \boldsymbol{\delta})=\sum_{\ell=1}^{v_{\text {exp }}} \log \left(p_{J}\left(J_{\ell}^{\text {exp }} ; \underline{\mathbf{r}}, \boldsymbol{\delta}\right)\right)
$$

The optimal value $(\underline{\mathbf{r}}, \boldsymbol{\delta})^{\mathrm{opt}}$ in $\mathfrak{R} \times \Delta$ is the solution of the following optimization problem,

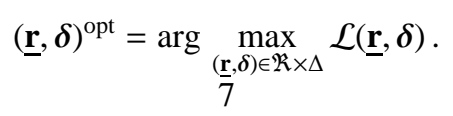


The calculation of $p_{J}\left(J_{\ell}^{\exp } ; \underline{\mathbf{r}}, \boldsymbol{\delta}\right)$ is done using the Monte Carlo simulation method (Rubinstein and Kroese, 2008) and the Gaussian kernel density estimation method (Bowman and Azzalini, 1997).

The optimal values of the prior probability distributions are $\underline{\mathbf{r}}^{\text {opt }}=(33208,0.0108,0.0024)$ and $\boldsymbol{\delta}^{\text {opt }}=(0.001,0.2658)$. Figure 3 displays the confidence region at room temperature for a probability level $P_{\mathrm{c}}=0.95$.

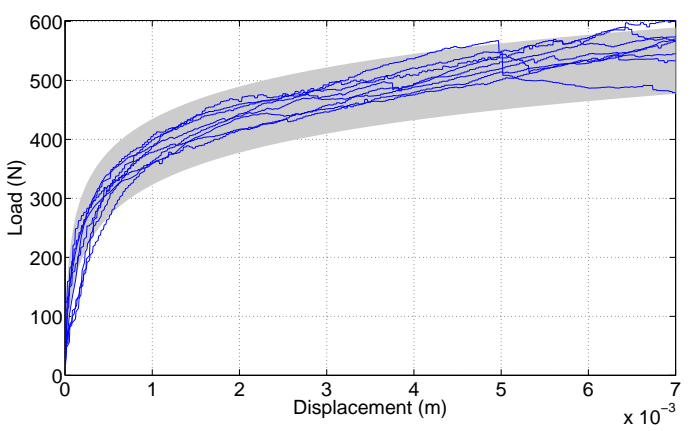

Figure 3: Comparison of the 8 experimental curves (eight solid lines) with confidence region calculated with the identified prior probability model (grey region) at room temperature.

For the high temperatures corresponding to 900,1200 and $1500 \mathrm{~s}$ of the thermal loading by the TLB, the identified parameters are summarized in Table 1. Figures 4 to 6 display the confidence region of thermal loading at the different moments for a probability level $P_{\mathrm{c}}=0.95$. For a significant displacement (for instance for $x=0.004 \mathrm{~m}$ ), the load decreases when the temperature increases while the variability increases. It should also be noted that $\delta_{A}$ is almost zero while $\delta_{B}$ is increasing with the temperature.

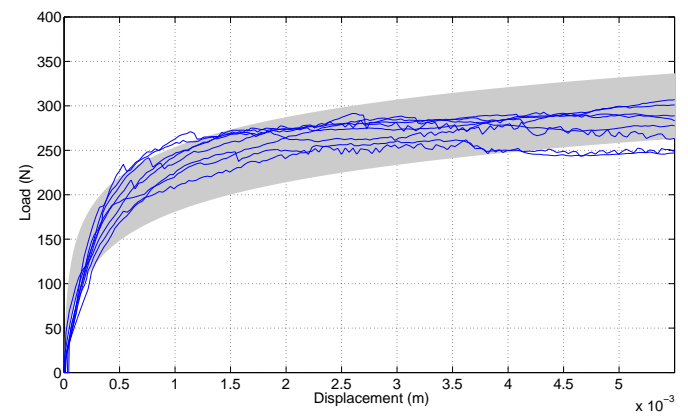

Figure 4: Comparison of the 8 experimental curves (eight solid lines) with confidence region calculated with the identified prior probability model (grey region) at $900 \mathrm{~s}$ of thermal loading. 


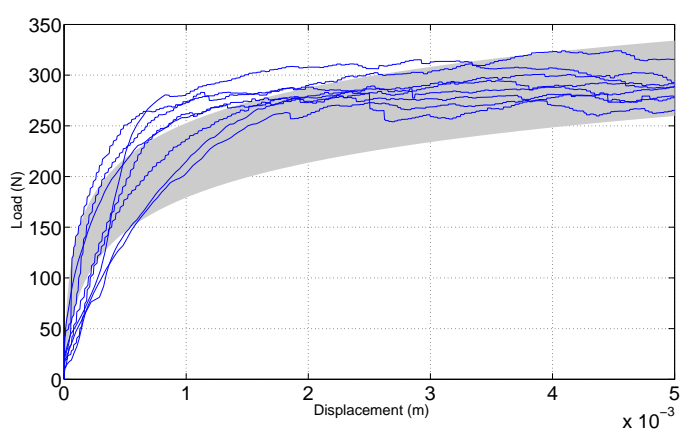

Figure 5: Comparison of the 8 experimental curves (eight solid lines) with confidence region calculated with the identified prior probability model (grey region) at $1200 \mathrm{~s}$ of thermal loading.

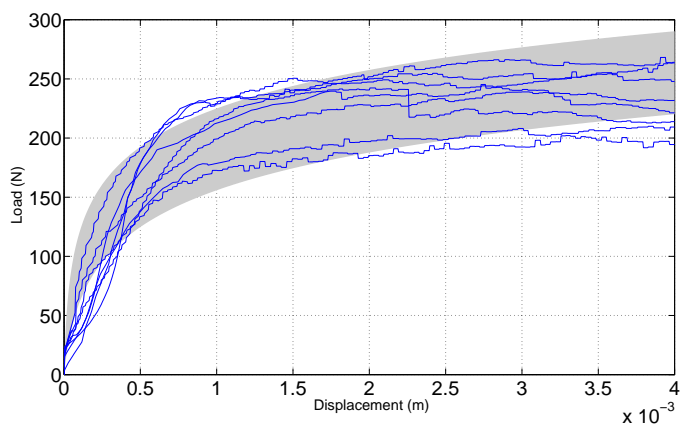

Figure 6: Comparison of the 8 experimental curves (eight solid lines) with confidence region calculated with the identified prior probability model (grey region) at $1500 \mathrm{~s}$ of thermal loading.

\section{EXPERIMENTAL IDENTIFICATION OF THE THERMAL AND THERMOMECHAN- ICAL CHARACTERISTICS OF A CPC PLATE}

For a complete mechanical/computational model of the light partition wall, it is necessary to determine the thermal and thermomechanical characteristics of the CPC plates, subjected to the reference thermal loading defined by the ISO 834 curve. These characteristics depend on the rate of rise in temperature.

\subsection{Experimental measurements of the thermal dilatation and of the thermal curvature}

Ten rectangular CPC plates (the samples) with dimension $0.1 \mathrm{~m}$ by $0.4 \mathrm{~m}$ are subjected to the thermal loading on one side, induced by the TLB. The thermal dilatation and the thermal curvature of the 10 tests are estimated and presented in Figures 7 and 8.

\subsection{Experimental measurements of the mass loss and of the temperature for the CPC plates}

The tests are designed to measure (1) the temperature of the exposed and unexposed sides, (2) the evolution of the mass of the CPC plates subjected to the thermal loading delivered by the TLB. For these tests, we used the samples with the same size as those of thermal deformation tests 


\begin{tabular}{cccccc}
\hline \hline Time & $\underline{a}^{\text {opt }}$ & $\underline{b}^{\text {opt }}$ & $\alpha^{\text {opt }}$ & $\delta_{A}^{\text {opt }}$ & $\delta_{B}^{\text {opt }}$ \\
\hline $0 \mathrm{~s}$ & 33208 & 0.0108 & 0.0024 & 0.001 & 0.2658 \\
$900 \mathrm{~s}$ & 33101 & 0.0152 & 0.0018 & 0.001 & 0.2730 \\
$1200 \mathrm{~s}$ & 32684 & 0.0184 & 0.0017 & 0.001 & 0.2756 \\
$1500 \mathrm{~s}$ & 31995 & 0.0212 & 0.0015 & 0.001 & 0.2810 \\
\hline
\end{tabular}

Table 1: Identified parameters of the prior probability distributions for the shear behavior of the screw attachment with a single skin at room and high temperatures.

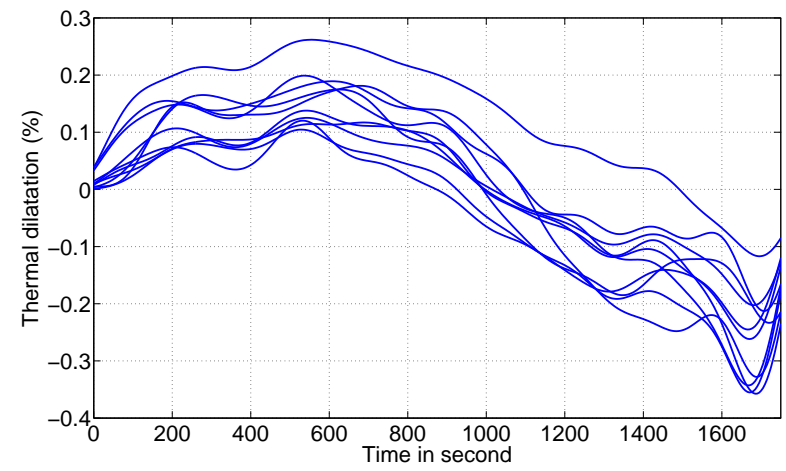

Figure 7: Thermal dilatation of the CPC plate versus time of thermal loading delivered by the TLB. The 10 curves represent the results of the 10 tests.

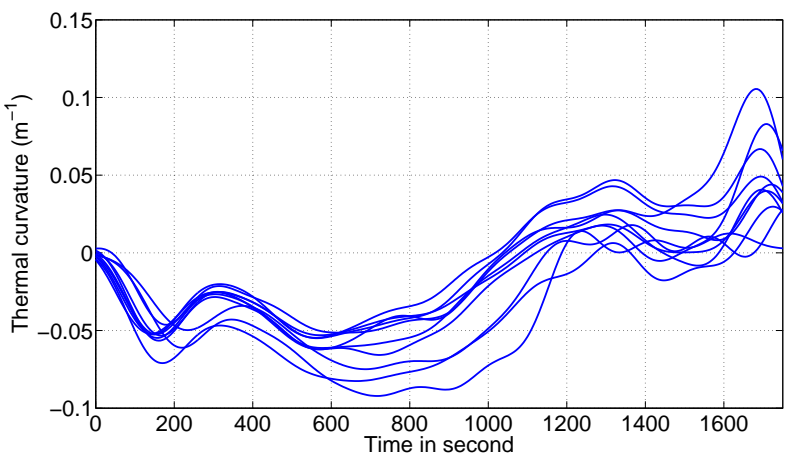

Figure 8: Thermal curvature of the CPC plate at about $1800 \mathrm{~s}$ of thermal loading delivered by the TLB. The 10 curves represent the results of the 10 tests.

presented in Section 3.1. Figure 9 shows the measured temperatures for 5 CPC plates. Figure 10 displays the measured mass loss for 5 other ones.

\subsection{Global analysis of the experimental measurements for the thermal deformation, the temperature and the mass loss}

By observing and analyzing the deformation measurements, the temperature and the mass loss tests of the CPC plates subjected to the thermal loading delivered by the TLB, the thermal be- 


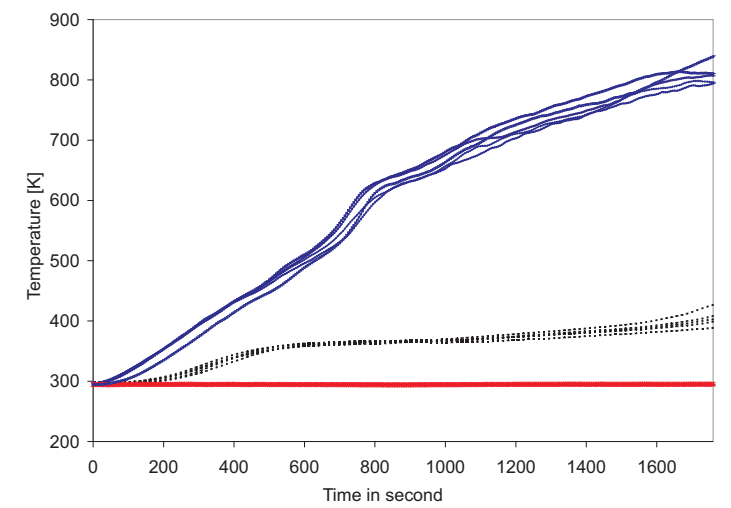

Figure 9: Temperature for each of the two sides of the CPC plates. The continuous thin lines are the temperatures on the exposed sides, the dashed lines are the temperatures on the unexposed sides and the continuous thick line represents the ambient temperature.

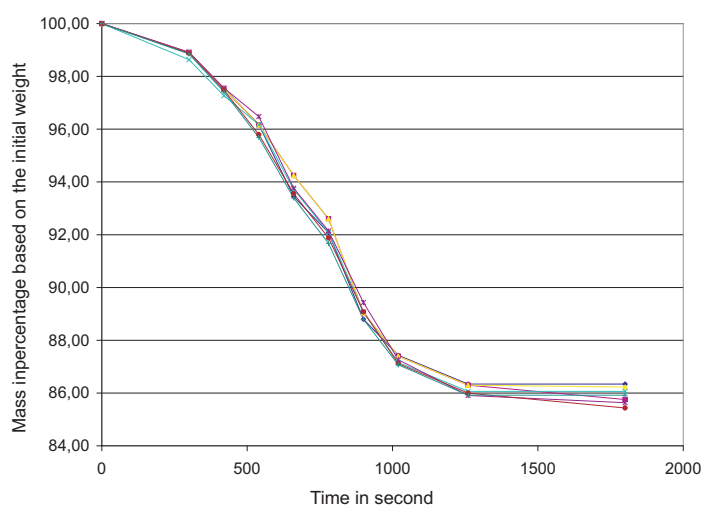

Figure 10: Mass loss in percentage based on the initial weight

havior of the CPC plates can be decomposed into two main stages: the thermal dilatation and the water withdrawal.

Thermal dilatation stage. In the first $300 \mathrm{~s}$, the phenomenon of water migration is dominant and the temperature of water increases. This increase produces a strong thermal dilatation of the CPC plates (the mass loss is very small in this phase). Between 300 and $420 \mathrm{~s}$, a strong increase of temperature on the unexposed side of the CPC plate occurs. The slope of the curve of evolution of the mass loss also increases. The water near the exposed surface of the plaster layer evaporates. During this period, the thermal dilatation (homogenized on the thickness of the CPC plates) is almost unchanged. Then, from 420 to $600 \mathrm{~s}$, the rate of increase of the temperature on the unexposed side decreases. The phenomenon of water evaporation is dominant. From $600 \mathrm{~s}$, the thermal dilatation reaches its maximum value. Then, the CPC plates are contracting in their plan. The temperature on the unexposed sides remains almost constant until $1080-1200 \mathrm{~s}$. In this period, the mass loss is regular. After $1080-1200 \mathrm{~s}$, the length and the width of the CPC plates return to their initial values. The water evaporation is no longer observed. This fact is con- 
firmed by the decrease of the rate of the mass loss. The behavior of the CPC plates is modified and the water withdrawal starts.

Water withdrawal stage. After about 1080 - $1200 \mathrm{~s}$, the rate of increase of the temperature on the unexposed side increases while the rate of the evolution of the mass loss is almost zero because the water in the plaster is almost completely evaporated and then the phenomenon of dilatation is reversed. The CPC plates are contracting in their plan.

\subsection{Tensile test}

The objective of these tests is to give the time evolution of the tension behavior of the CPC plates subjected to the thermal loading according to ISO 834 curve delivered by the TLB. The mechanical tensile tests are carried out using a uniaxial tension bench (for the details, see Do (2011).

Figures 11, 12 and 13 show the results for the test specimens at room temperature and at high temperature after 900 and $1200 \mathrm{~s}$ of the thermal loading. It is noted that the ordinates of these figures begin from $-10^{5} \mathrm{~Pa}$ due to the prestressing. There is a strong evolution of the tensile

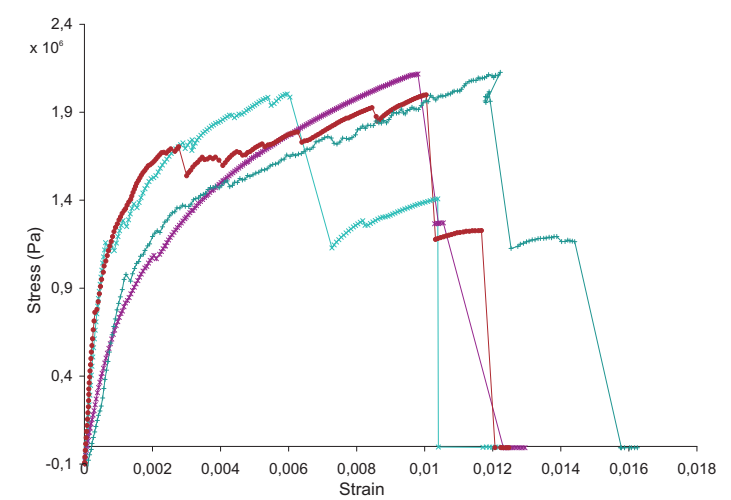

Figure 11: Stress-strain curves for 5 samples, in tension, at room temperature. Stress in $\mathrm{Pa}$ as a function of the strain.

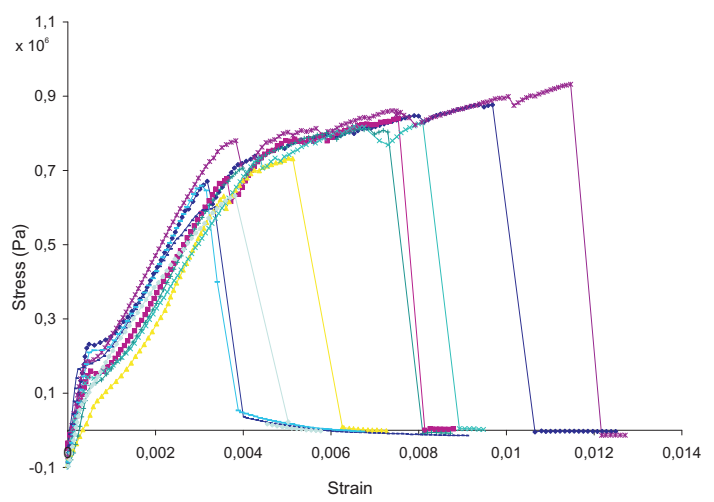

Figure 12: Stress-strain curves for 7 samples, in tension, after $900 \mathrm{~s}$ of thermal loading. Stress in Pa as a function of the strain. 


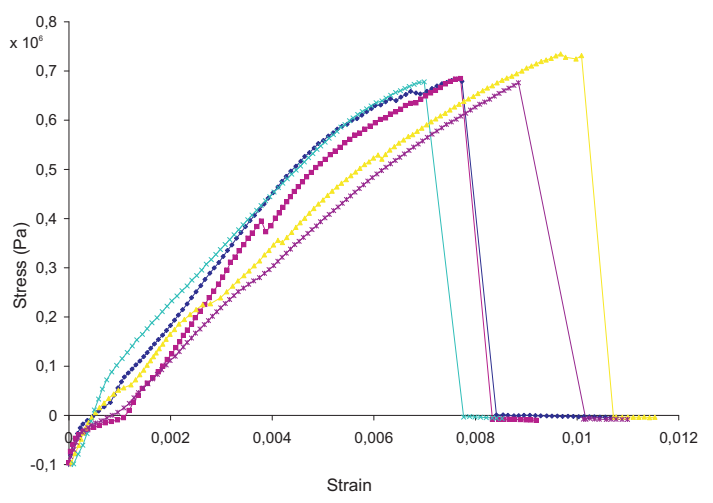

Figure 13: Stress-strain curves for 5 samples, in tension, after $1200 \mathrm{~s}$ of thermal loading. Stress in Pa as a function of the strain.

behavior of the CPC plates as a function of the temperature. There is a high dispersion of the limit values of the deformation corresponding to the tensile breaking limit, at the same instant of the thermal loading for the CPC plates that should be the same. The tensile tests of the CPC plates subjected to the thermal loading are particularly difficult to realize and have been conducted very carefully and with adapted methodologies. These measurements are described in detail in (Do, 2011). The large variability experimentally observed is not due to measurements errors but are due to the type of materials (cardboard and plaster) and to the manufacturing processes of the CPC plates.

\subsection{Compression test}

The compression tests of the CPC plates are carried out under the thermal loading according to ISO 834 delivered by the TLB. The mechanical compression tests are carried out using a tensile bench adapted to generate a compression. The compression tests are performed until a limit of deformation measured in the range of $4 \%$ (in practice, the partition walls with CPC plates do not work beyond $4 \%$ of deformation) (for additional explanations, the reader is referred to Do (2011).

Figures 14 and 15 to 17 show the experimental results for the test specimens at room temperature and after 900, 1200 and $1500 \mathrm{~s}$ of the thermal loading delivered by the TLB. There is a strong evolution of the compression behavior as a function of temperature. For a given instant of the thermal loading, there is a greater dispersion of the compression behavior than for the tension behavior. Similarly to the tensile tests, the compression tests of the CPC plates subjected to the thermal loading are also difficult to realize. These measurements are described in detail in (Do, 2011). The sources of variability are always due to the materials (cardboard and plaster) and to the manufacturing processes of the CPC plates.

\section{SEMI-ANALYTICAL MODEL FOR CALCULATING THE DEFLECTION OF A LARGE LIGHT PARTITION WALL UNDER A DETERMINISTIC UNIFORM LOAD}

This section deals with the probabilistic analysis of the deflection for a large light partition wall under a deterministic uniform load. The method of calculation is based on the use of a mechanical model made up of beams and plates connected by screws (see Figure 18) and submitted to an 


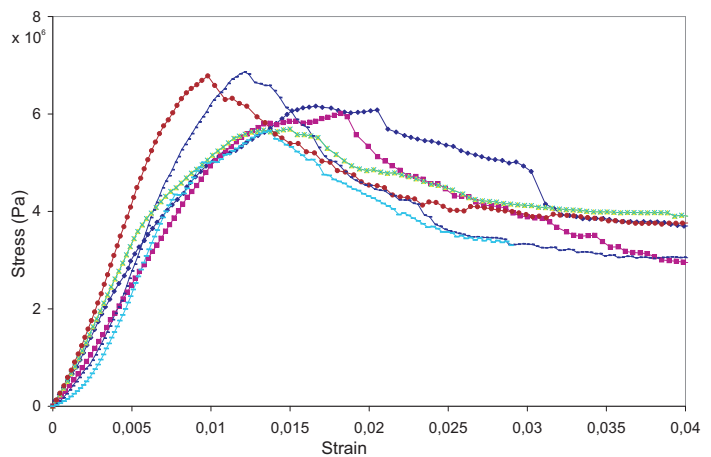

Figure 14: Stress-strain curves for 6 samples, in compression, at room temperature. Stress in Pa as a function of the strain.

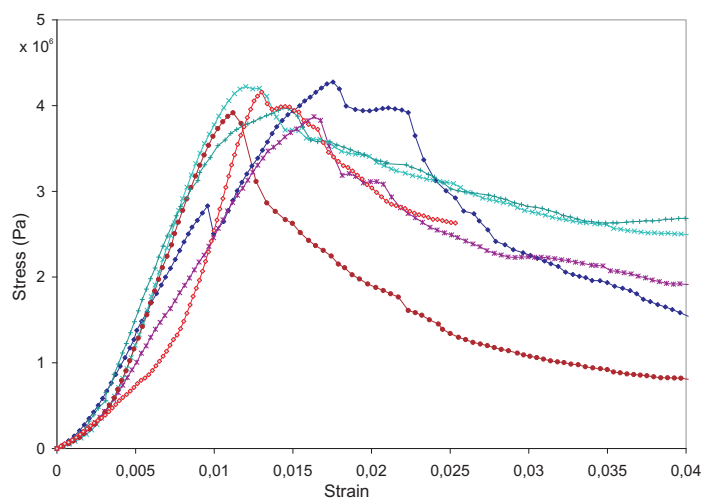

Figure 15: Stress-strain curves for 6 samples, in compression, after $900 \mathrm{~s}$ of thermal loading. Stress in Pa as a function of the strain.

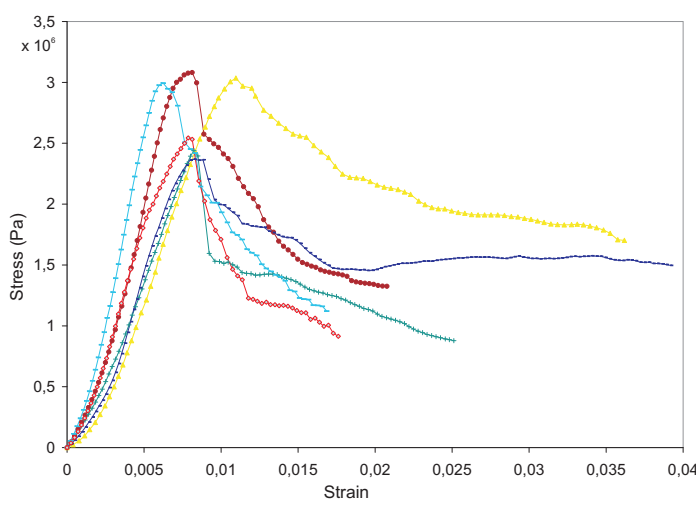

Figure 16: Stress-strain curves for 6 samples, in compression, after $1200 \mathrm{~s}$ of thermal loading. Stress in Pa as a function of the strain.

out-of-plane uniform static load ( $x_{3}$-axis) applied to the plates. It is assumed that, for the service limit state, the large light partition wall works in the linear elastic domain. This hypothesis is 


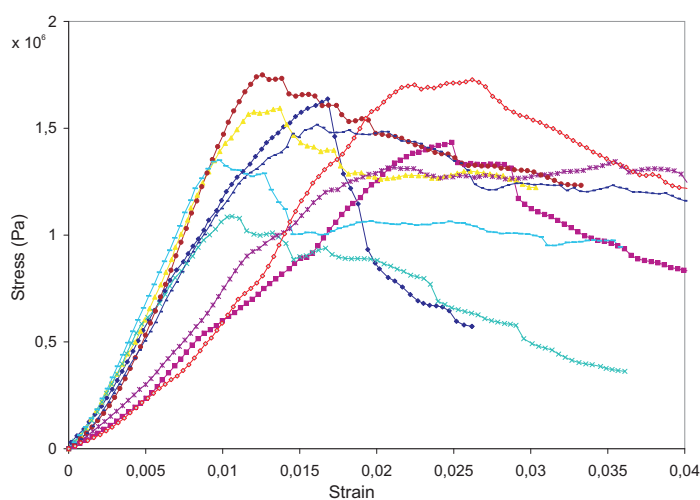

Figure 17: Stress-strain curves for 8 samples, in compression, after $1500 \mathrm{~s}$ of thermal loading. Stress in Pa as a function of the strain.

then retained in the analysis presented below for the beams and plates but not for the screws which have a nonlinear behavior. The mechanical model of the large light partition wall uses the

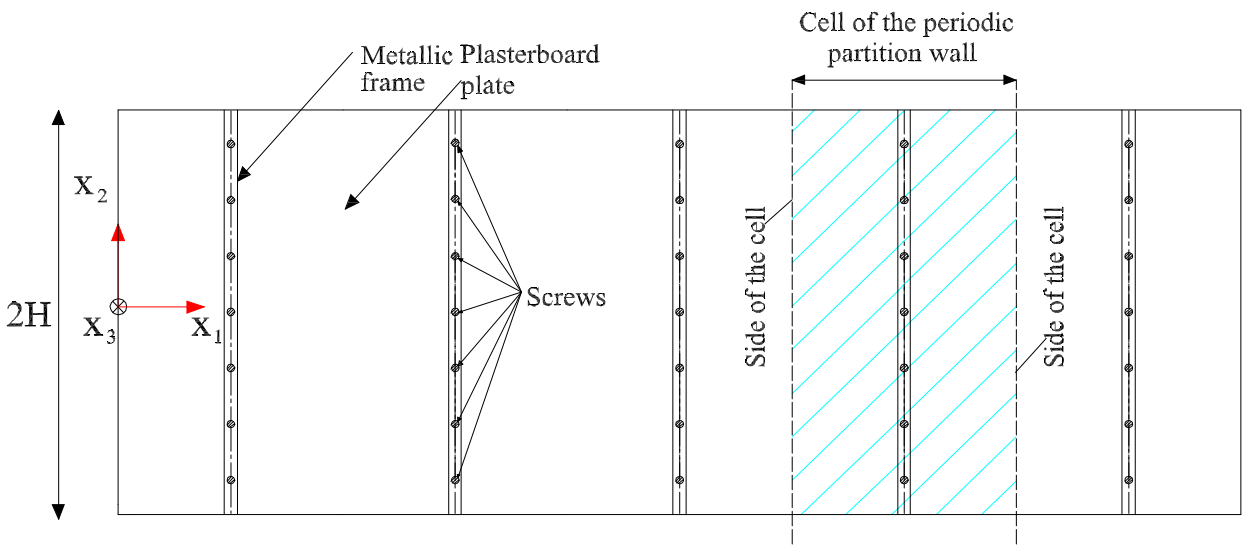

Figure 18: Large light partition wall submitted to an out-of-plane uniform static load.

following kinematic hypotheses: (1)- The large light partition wall is considered as a periodic structure for which the cell is shown in Figure 18. Consequently, the calculation is carried out using the cell with periodic boundary conditions for which the $x_{1}$-displacements are zero on the two sides of the cell (see Figure 18). (2)- The metallic frame of the cell is modeled by a Bernoulli beam, in bending mode for the out-of-plane displacements. (3)- The plate is represented by a mixed kinematic of the Bernoulli beam type for bending mode and of the plate-theory type for membrane mode. (4)- A discontinuity of the in plane displacements is introduced at the level of the screws in order to represent the connection between the CPC plates and the metallic frame. This discontinuity is made possible by the rotation of screws around the $x_{1}$-axis. The mechanical model of the cell of the large light partition wall is shown in Figure 19. The deflection $\left(x_{3^{-}}\right.$ 

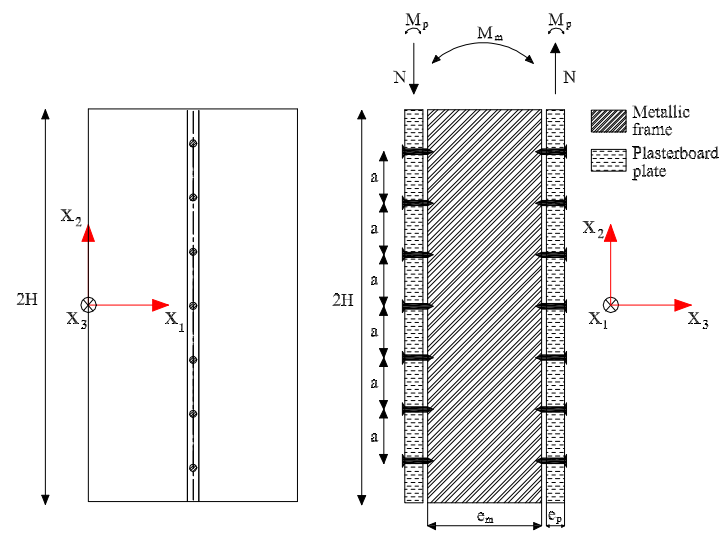

Figure 19: Representative section of the cell for the large light partition wall.

displacement) at the middle point of the cell is given by

$$
W=\frac{5 p H^{4}}{24 R}-\frac{a}{R} \sum_{i=1}^{n} i\left(H-\frac{i a}{2}\right)\left(e_{m}+e_{p}\right) Y_{i},
$$

in which, $W$ is the deflection ( $x_{3}$-displacement) at the middle point of the cell; $p$ is the linear load corresponding to the uniform load multiplied by the distance between two consecutive metallic frames (the width of the representative cell); $2 H$ is the height of the large light partition wall; $R$ is the main bending stiffness such that $R=E_{m} I_{m}+2 E_{p} I_{p}$ in which $E_{m}$ and $I_{m}$ are the modulus and the inertia of the metallic frame and where $E_{p}$ and $I_{p}$ are those for the plates; $a$ is distance between two consecutive screws; $e_{p}$ and $e_{m}$ are the thicknesses of the plate and of the metallic frame; $n$ is the number of screws on a half-height $H$ of the large light partition; for $i=1, \ldots, n$, $Y_{i}$ is the force applied to the $i-t h$ screw. The first term in the right-hand side of Equation (18) is the deflection which would be obtained if the plate and the metallic frame were simply plated to each other without screw. The second term expresses the decrease of the deflection caused by the screws. The relation between applied forces $Y_{i}$ and displacement $X_{i}$ on the $i^{\text {th }}$ screw has been studied in the previous sections and is described by a probabilistic constitutive equation. It could be rewritten by introducing the stiffness modulus $K\left(X_{i}\right)$ as

$$
Y_{i}=A_{i}\left[\left(X_{i}+B_{i}\right)^{\alpha}-B_{i}^{\alpha}\right]=X_{i} K\left(X_{i}\right) .
$$

The random equations are solved by the Monte Carlo method. Consequently, for each screw, the independent realizations of random variables $A$ and $B$ are constructed with the generators corresponding to the probability density functions defined by Equations (10) and (11). The random displacements $X_{1}, \ldots X_{n}$ of the screws are given by solving, for all $i$ in $1, \ldots, n$, the system of random equations,

$$
\sum_{j=1}^{n}\left\{\frac{2 R \delta_{i j}}{e_{m}+e_{p}}+\eta_{i j} K\left(X_{j}\right)\right\} X_{j}=\frac{p H^{3}}{6}\left(3 \frac{i a}{H}-\left(\frac{i a}{H}\right)^{3}\right),
$$

with $\eta_{i j}=2 R d(j a, i a) /\left\{E_{p} e_{p}\left(e_{m}+e_{p}\right)\right\}+\min (i, j) a\left(e_{m}+e_{p}\right)$ in which $\delta_{i j}=0$ if $i \neq j$ and $=1$ if $i=j$, and where $d(j a, i a)$ is the membrane displacement in $x_{2}$ at the height level $x_{2}=i a$ of 
the plasterboard plate which would have a unit thickness and a unit modulus and which would be submitted to a unit load at the height level $x_{2}=j a$. The large light partition wall is such that, $2 H=2.7 \mathrm{~m}, p$ is a linear load corresponding to the dimensional uniform pressure $200 \mathrm{~N} / \mathrm{m}^{2}, a=$ $0.3 \mathrm{~m}, e_{p}=0.0125 \mathrm{~m}$ and $e_{m}=0.05 \mathrm{~m}$. The value $w_{0}$ of the deflection $W$ calculated with the identified nominal model is $w_{0}=0.0056 \mathrm{~m}$. The mean-square convergence of the Monte Carlo method is reached with 2,500 realizations. The estimation of the probability density function of the random deflection $W$ at the middle of the large light partition wall is given in Figure 20. The mean value and the standard deviation of the random deflection are $\underline{W}=0.0054 \mathrm{~m}$ and $\sigma_{W}=$ $0.00014 \mathrm{~m}$. The prediction of $W$ given by the nominal model which is $w_{0}=0.0056 \mathrm{~m}$ is near

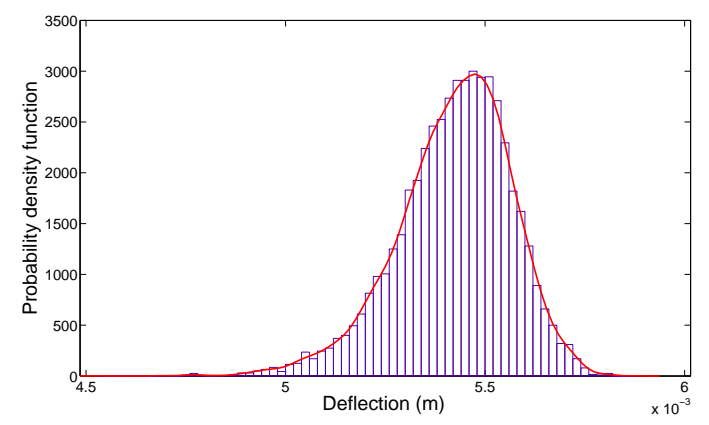

Figure 20: Graph of the probability density function of the random deflection $W$ at the middle height of the large light partition wall submitted to a uniform load.

the mean value $\underline{W}=0.0054 \mathrm{~m}$ of the random response given by the probabilistic model, and the dispersion is relatively small. For 10,000 realizations and for each of the 4 screws (located at the middle and the three upper screws), the minimum force and the maximum force are given in Table 2 .

\begin{tabular}{ccc}
\hline \hline $\begin{array}{c}\text { Screw } \\
\text { number }\end{array}$ & $\begin{array}{c}\text { Minimum } \\
\text { force }(\mathrm{N})\end{array}$ & $\begin{array}{c}\text { Maximum } \\
\text { force }(\mathrm{N})\end{array}$ \\
\hline 1 & 88 & 313 \\
2 & 149 & 376 \\
3 & 180 & 479 \\
4 & 198 & 466 \\
\hline
\end{tabular}

Table 2: Minimum and maximum forces in $\mathrm{N}$ for each of the 4 screws with 10,000 realizations

Table 2 shows that there are significant statistical fluctuations of the forces in the screws, but these fluctuations induce relatively weak statistical fluctuations for the sliding. This is due to the statistical averaging effect with respect to the number of screws and consequently, which reduces the statistical fluctuations of the random deflection $W$. Therefore, such a result allows the design to be optimized in a robust framework with respect to variability and to uncertainties. 


\section{NONLINEAR THERMOMECHANICAL COMPUTATIONAL MODEL FOR A LARGE LIGHT PARTITION WALL}

This section deals with the presentation of a nonlinear thermomechanical model for a large light partition wall subjected to thermal loading according to ISO 834 curve and under the effect of a uniform distributed load. To describe the behavior of the partition, the following kinematic choices are made:

- The CPC plates are modeled by the thick plate theory.

- The metallic frames ("C" profile) are modeled by Bernoulli thin open section beams.

- The screw attachments between the CPC plates and the metallic frame are modeled by a springs system with nonlinear behaviors for which the stiffnesses are calculated using the shear behavior model of the screw attachment presented and experimentally identified in Section 2.

- The mechanical model used to represent the shear force transmitted by a screw between a CPC plate and the metallic frame is punctual (the force is concentrated at one point). Such a model would cause a significant local deformation in the CPC plate and would not correspond to the physical reality. Consequently, in the computational model, this force is distributed locally in the vicinity of the screw. To better present this reality and the diffusion of applied force by the screw in the CPC plate, we introduced an undeformable subdomain (disc shaped), centered on the screw with a radius $r=0.015 \mathrm{~m}$. Thus, the concentrated force at position of the screw is distributed on the circumference of this subdomain, so ensuring distribution of force in the CPC plate.

For the model of the screw attachment in the computational model, the attachment is modeled by a connecting two nodes element, one node is located on the neutral axis of the metallic frame and the other one is located on the medium plane of the CPC plate. The elementary stiffness matrix of this element is constructed by an energetic approach (Do, 2011).

\section{SIMULATION OF A LARGE LIGHT PARTITION WALL}

In this section, we present the simulation of the mechanical behavior at room temperature and the thermomechanical behavior at a given high temperature of a large light partition wall for which the cell consists of CPC plates screwed on the metallic frame (see Figure 19). A uniform pressure field is applied to the CPC plate on the side which is not exposed to the thermal loading. All the results are obtained using a home software for nonlinear thermomechanical calculations, developed by CSTB (Centre Scientifique et Technique du Bâtiment), in which the following ingredients have been implemented:

1. The laws of the deterministic and probabilistic shear behaviors of the screw attachments, at room temperature and at high temperature under thermal loading.

2. The plate finite element, homogenized in thickness, for the CPC plates, and the Bernoulli beams with thin open section, for which the hypotheses are given in Section 5.

3. The stochastic solver based on the use of the Monte Carlo method and the statistical estimators for solving the stochastic computational model related to the probabilistic modeling of the screw attachments.

The simulations are carried out for a large light partition wall on the cell with periodic boundary conditions. The cell consists of two standard CPC plates, each one with $0.6 \mathrm{~m}$ width (the distance between two metallic frames is $0.6 \mathrm{~m}$ ), $2.7 \mathrm{~m}$ height (height of the large light partition wall), 
$e_{p}=0.0125 \mathrm{~m}$ thickness. The distance between two screw along the longitudinal axis of the metallic frame is $a=0.3 \mathrm{~m}$. The section of the metallic frame has a profile in "C" with $0.048 \mathrm{~m}$ width, $0.05 \mathrm{~m}$ height and $0.006 \mathrm{~m}$ thickness.

\subsection{Simulation of the mechanical behavior with the nominal computational model at room temperature}

The cell is subjected to a uniform pressure of $200 \mathrm{~N} / \mathrm{m}^{2}$. For the CPC plate, the experimental measurements are used for the Young modulus and the Poisson ratio of the homogenized CPC plate. The experimental Young modulus is estimated from Figure 11 and is $2 \times 10^{9} \mathrm{~Pa}$. The experimental Poisson ratio is the value 0.16 given in Sakji (2006). The metallic frame is in steel, with $2.1 \times 10^{11} \mathrm{~Pa}$ for the Young modulus and 0.2 for the Poisson ratio. For the screw attachment, the model parameters of shear behavior at room temperature (see Section 2.4 and Equation (7) are such that $\left(a_{0}, b_{0}, \alpha_{0}\right)=(32108,0.01905,0.00294)$, these coefficients having dimensions such as the displacement $x_{0}$ is in millimeter $\left(10^{-3}\right.$ meter $)$ and the applied load $y_{0}$ is in Newton. Figure 21 represents the displacement field out of plane (deflection field in bending mode) of the cell.

At the middle height of the large light partition wall, the deflection calculated with the nominal
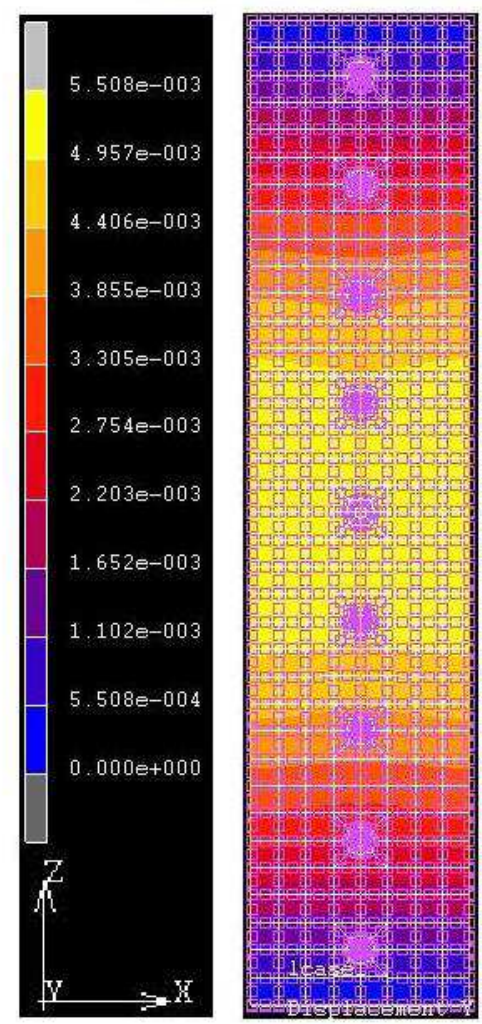

Figure 21: Displacement out of plane of the cell subjected to the uniform pressure given by the nominal model at room temperature.

computational model is $0.0055 \mathrm{~m}$ which is close to $0.0056 \mathrm{~m}$ of the deflection calculated by using 
the semi-analytical model presented in Section 4.

\subsection{Simulation of the mechanical behavior with the stochastic computational model at room temperature}

The simulation is performed using the stochastic computational model presented in Section 5. For the CPC plates and the metallic frame, the data are those used in Section 6.1. For the screw attachments and for time $0 \mathrm{~s}$, the optimal values of the parameters are given in Table 1 and are $\left(\underline{a}^{\mathrm{opt}}, \underline{b}^{\mathrm{opt}}, \underline{\alpha}^{\mathrm{opt}}, \delta_{A}^{\mathrm{opt}}, \delta_{B}^{\mathrm{opt}}\right)=(33208,0.0108,0.0024,0.001,0.2658)$, the parameters $\left(\underline{a}^{\mathrm{opt}}\right.$ and $\underline{b}^{\mathrm{opt}}$ having dimensions such that the displacement $X$ is in millimeter $\left(10^{-3}\right.$ meter) and the force $Y$ is in Newton. The convergence is reached for $n_{s} \geq 800$. The calculations are performed with $n_{s}=1000$. The probability density function of the random deflection $W$ at the middle height of the large light partition wall, is computed using the Gaussian kernel density estimation method (in the context of nonparametric statistics) and is shown in Figure 22. At the middle height of the large light partition wall, the deflection calculated with the nominal computational model, which is $0.0055 \mathrm{~m}$, is close to the statistical mean value $\underline{W}=0.00576 \mathrm{~m}$ of the random deflection given by the stochastic computational model. The estimation of the standard deviation $\sigma_{W}$ of the random deflection $W$ is $\sigma_{W}=0.000068 \mathrm{~m}$. Figure 22 shows that the probability distribution of the random variable $W$ is not Gaussian. By comparing the results provided by the stochastic semi-analytical model with the results given by the stochastic computational model, a difference can be seen on the second-order moments, that is to say, on the statistical mean value $0.00576 \mathrm{~m}$ which must be compared to $0.00540 \mathrm{~m}$, and on the standard deviation $0.000068 \mathrm{~m}$ which must be compared to $0.00014 \mathrm{~m}$. Finally, the probability density function calculated with the stochastic computational model is presented in Figure 22) which has to be compared to Figure 20. It should be noted that the results given by the stochastic computational model are converged with respect to the finite element mesh and to the number of simulations for the Monte-Carlo method. The differences given by the two predictions can be explained by differences in the mechanical modeling, particularly by those used to represent the transfer of the shear forces exerted by the screws in the CPC plates. For the computational model, the transfer of the shear forces exerted by the screws in the CPC plates is modeled by introducing an undeformable domain around the axis of each screw. In the semi-analytical model, simplified hypotheses are introduced. In the absence of experimental results for the large light partition wall which is modeled, it is not possible to say what would be the best prediction. Anyway, the stochastic semi-analytical model and the stochastic computational model yield similar results. Both the models give close statistical mean deflections and very small standard deviations. The small standard deviation of the deflection can probably be explained by the fact that, although the dispersion of the shear forces of the screw attachments is relatively large, the presence of a large number of screw attachments (18 for the cell) induces an effect of space averaging in the response (random deflection), and consequently, induces a small dispersion of the random deflection.

\subsection{Simulation of the thermomechanical behavior with the stochastic computational model at high temperature under thermal loading}

Simulation of the heat transfer for the large light partition wall. The simulation of heat transfer of the large light partition wall is carried out on the cell with periodic boundary conditions, using SAFIR (Franssen, 2005), available at CSTB. The thermophysical properties used for the plaster are those given by Mehaffey and Cuerrier (1994) and Do (2011), and the thermophysical properties for the metallic frame are those given by Eurocode 3. It should be noted that, for 


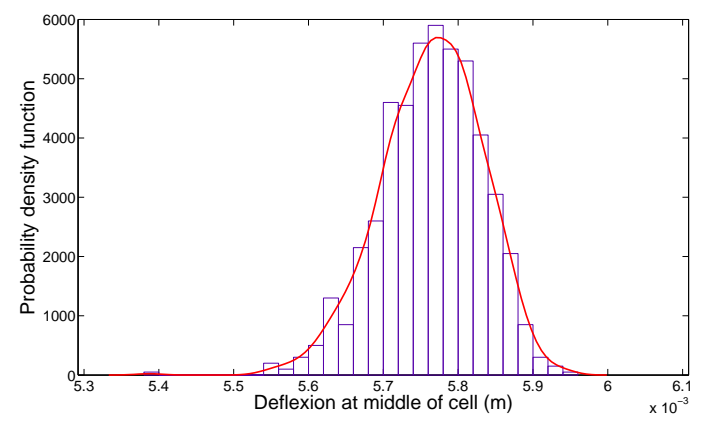

Figure 22: Graph of the probability density function of the random deflection $W$ at the middle height of the large light partition wall.

the heat transfer model, the cardboard sheets (which play an important role for the mechanical behavior) are not taken into account for the heat transfer. At $1200 \mathrm{~s}$ of the thermal loading, the temperature field in the section of the cell is displayed in Figure 23. The upper part of Figure 23

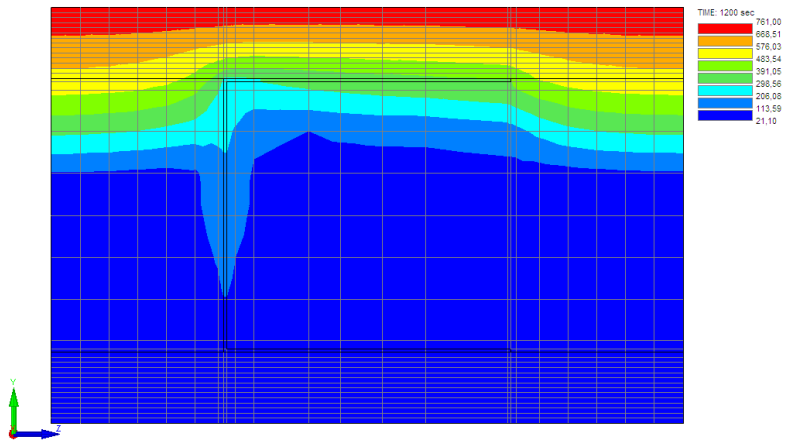

Figure 23: Temperature field in the section of the cell at $1200 \mathrm{~s}$ of thermal loading. The upper part of the figure corresponds to the exposed side to fire. Le temperature levels are in Celcius and the different color levels from the red to the blue color correspond to the following values: $761,668,576,483,391,298,208,113$ and $21^{\circ} \mathrm{C}$

corresponds to the exposed side to fire. The temperature in the neighborhood of the exposed side is about $760{ }^{\circ} \mathrm{C}$ while the temperature on the unexposed side (lower part of Figure 23) remains at room temperature $21^{\circ} \mathrm{C}$. Approximately, the lower two thirds, remains at room temperature with the exception of the area surrounding the metallic profile for which the thermal conduction is important. At this time of the thermal loading, the thermal gradient is very large in the upper third of the cell.

Simulation of the thermomechanical behavior with the stochastic computational model under thermal loading. The simulation of the thermomechanical behavior of the large light partition wall is performed with the stochastic computational model at high temperature corresponding to $1200 \mathrm{~s}$ of the thermal loading. At this high temperature, the experimental Young modulus and Poisson ratio are used for the homogenized CPC plate. The experimental Young 
modulus $1.2 \times 10^{8} \mathrm{~Pa}$ is deduced from Figure 13 and the experimental Poisson ratio 0.16 is given in Sakji (2006). The Young modulus and the Poisson ratio for steel of the metallic frame are given by Eurocode 3. For the screw attachments on the side exposed to fire, the parameters of the shear behavior of the screw attachments are given by Table 1, that is to say, $\left(\underline{a}^{\mathrm{opt}}, \underline{b}^{\mathrm{opt}}, \underline{\alpha}^{\mathrm{opt}}, \delta_{A}^{\mathrm{opt}}, \delta_{B}^{\mathrm{opt}}\right)=(32684,0.0184,0.0017,0.001,0.2756)$. Parameters $\left(\underline{a}^{\mathrm{opt}}\right.$ and $\underline{b}^{\mathrm{opt}}$ have dimensions such as the displacement $X$ is in millimeter $\left(10^{-3}\right.$ meter) and the load $\bar{Y}$ is in Newton. For the screw attachments on the side unexposed to fire, the heat transfer at 1200 $\mathrm{s}$ shows that the temperature of the screw attachments are at room temperature. Consequently, the previous data corresponding to the room temperature are used for the parameters of the shear behavior of these screw attachments. The convergence is reached for $n_{s} \geq 800$ independent realizations in the Monte Carlo method. The calculations are done with $n_{s}=1000$. The probability density function of the random deflection $W$ at the middle height of the large light partition wall (estimated as above) is shown in Figure 24. The statistical mean value of the random deflection $W$ given by the stochastic computational model is $\underline{W}=0.0619 \mathrm{~m}$, which corresponds to more than ten times the nominal model of the deflection at room temperature. The estimated standard deviation $\sigma_{W}$ of $W$ is $\sigma_{W}=0.0002 \mathrm{~m}$. This standard deviation is three times larger than for the random deflection calculated at room temperature. Figure 24 shows that the probability distribution of the random variable $W$ is not Gaussian. The comments introduced at the end of Section 6.2 concerning the level of statistical fluctuations of the random deflection at room temperature remain valid for this case at high temperature.

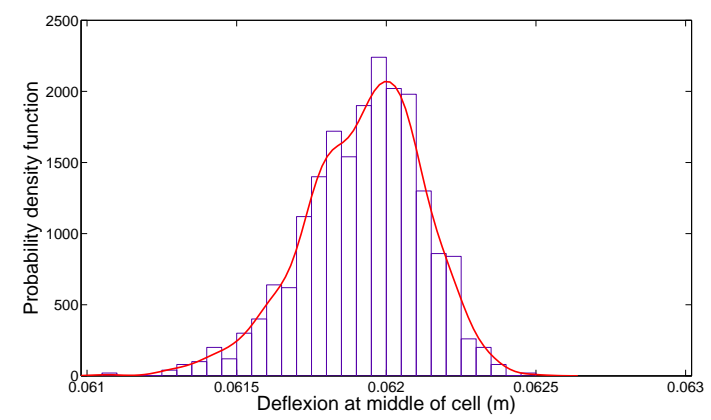

Figure 24: Thermal loading at $1200 \mathrm{~s}$. Graph of the probability density function of the random deflection $W$ at middle height of the large light partition wall.

\section{CONCLUSION}

In this paper, we have presented a research concerning the developments of a computational nonlinear thermomechanical model for the prediction of the behavior of large light partition walls made up of CPC plates screwed to metallic frames and submitted to mechanical load and to fire load. More precisely, a thermomechanical analysis of screwed plasterboard with nonlinear constitutive equation for screw attachment has been presented. This problem is very difficult and the alone use of a theroretical/computational approach cannot be done in the present state of the art, taking into account the complexity of the thermomechanical behavior of the CPC plates at high temperature and the nonlinear thermomechanical shear behavior of the screw attachments which depends on the temperature. Appropriate thermal and thermomechanical experiments have then 
been developed in the aim to construct a database for the CPC plates and for the screw attachments at room temperature and at high temperatures. This experimental database has allowed us to develop a semi-physical model for the nonlinear shear behavior of screw attachments and a computational nonlinear thermomechanical model for the large light partition walls submitted to mechanical loads and thermal loadings induced by fire. A major difficulty arise by the fact that the experimental results present a very important variability which has to be taken into account to develop a robust predictive computational model. This variability has been taken into account using a probabilistic approach for the shear behavior of the screw attachments. Based on the experimental database and on the developments of models, we have presented the simulation of the mechanical behavior of a large light partition wall at room temperature and its thermomechanical behavior at high temperature under mechanical and thermal loadings. A very interesting result has been exhibited concerning the small effects of the large variability of the shear forces in the screw attachments on the response in displacement of the large light partition wall for room temperature and for the high temperatures. The results presented allow the justification for the fire resistance of large light partition walls to be carried out with a robust model with respect to large variability of behavior of some components

\section{REFERENCES}

Andersson, L. \& Jacksson, B. (1995), Analytical Fire Design with Gypsum - A Theoretical and Experimental Study, Institute of Fire Safety Design, Lund, Sweden.

Axenenko, O. \& Thorpe, G. (1996), The modelling of dehydration and stress analysis of gypsum plasterboards exposed to fire, Computational Materials Science, 3(6), 281-294.

Beck, J. L. \& Katafygiotis, L. S. (1998), Updating models and their uncertainties. I: Bayesian statistical framework, Journal of Engineering Mechanics, 124(4), 455-461.

Beck, J. L., Graf, W. \& Katafygiotis, L. (2010), Special Issue on Computational intelligence in structural engineering and mechanics, Computer-Aided Civil and Infrastructure Engineering, 25(5), 303-393.

Benouis, A. (1995), Comportement mcanique des ouvrages en plaque de plpatre sur ossature mtallique, PhD Thesis, Ecole Nationale des Ponts et Chausses.

Bowman, A. W. \& Azzalini, A. (1997), Applied Smoothing Techniques for Data Analysis, Oxford University Press, Oxford, 1997.

Cachim, P. B. \& Franssen, J.-M. (2009), Numerical modelling of timber connections under fire loading using a component model, Fire Safety Journal, 44(6), 840-853.

Cheung, S. H. \& Beck, J. L. (2010), Calculation of the posterior probability for Bayesian model class selection and averaging from posterior samples based on dynamic system data,ComputerAided Civil and Infrastructure Engineering, 255, 304-321.

Cooper, L. Y. (1997), The Thermal Response of Gypsum-panel/steel-stud Wall Systems Exposed to Fire Environments: A Simulation for Use in Zone-type Fire Models, US Dept. of Commerce, Technology Administration, National Institute of Standards and Technology.

Cover, T.M. \& Thomas, J.A. (2006), Elements of Information Theory, Second Edition, John Wiley \& Sons, Hoboken.

Cramer, S. M., Friday, O. M., White, R. H. \& Sriprutkiat, G., (2003), Mechanical Properties of Gypsum Board at Elevated Temperatures, Proceeding of the Fire and Materials 2003, San Francisco, California, USA, pp. 27-28. 
Do, T. T. (2011), Experimental analysis and modeling of the nonlinear thermomechanical behavior of a large light partition wall made up plates plasterboard and cardboard, screwed and subjected to thermal and mechanical loads. PhD Thesis, Universit Paris-Est, France, 2011. The pdf file of the thesis is available on line at http://tel.archives-ouvertes.fr/tel-00665543/.

Franssen, J. M. (2005), SAFIR: A thermal/structural program for modeling structures under fire, Engineering Journal-American Institute of Steel Construction, 42(3), 143-158.

Fuller, J. J., Leichti, R. J. \& White, R. H. (1992), Temperature distribution in a nailed gypsumstud joint exposed to fire, Fire and materials, 16(2), 95-99.

Gerlich, J. T. (1995), Design of Loadbearing Light Steel Frame Walls for Fire Resistance, in Fire Engineering Research Report 95/3, University of Canterbury, Christchurch, New Zealand.

Goncalves, T., Jong, T., Clancy, P. \& Poynter, W. (1996), Mechanical Properties of Fire Rated Gypsum Board, Department of Civil and Building Engineering, Victoria University of Technology.

Hadjisophocleous, G. (1996), Extract from Report on Furnace Tests on Walls for Departement of National Defence, Canada, National Fire Laboratory, NRCC, National Research Council Canada. Harmathy, T. Z. (1988), Properties of building materials, National Research Council Canada, Institute for Research in Construction.

Irfanoglu, A. (2012), Using numerical simulations and engineering reasoning under uncertainty: Studying the collapse of WTC-1, Computer-Aided Civil and Infrastructure Engineering, 27(1), 65-76.

Jaynes, E. T. (1957a), Information theory and statistical mechanics, Physical Review, 106, 620630.

Jaynes, E. T. (1957b), Information theory and statistical mechanics II, Physical Review, 108(2), 171-190.

Jones, M. H. \& Wang Y. C. (2008), Experimental studies and numerical analysis of the shear behavior of thin plates to tubular columns at ambient and elevated temperatures, Steel And Composite Structures, 8(3), 179-200.

Kapur, J. N. \& Kesavan, H. K. (1992), Entropy optimization principles with applications, Academic Press, San Diego.

Kodur, V. K. R., Dwaikat, M. M. S. \& Dwaikat M. B. (2008), High-temperature properties of concrete for fire resistance modeling of structuresACI Materials Journal, 105(5), 517-527.

Konig, J. \& Walleij, L. (2000), Timber frame assemblies exposed to standard and parametric fires, Part 2: A Design Model for Standard Fire Exposure, Technical report - Institutet fr Trteknisk Forskning, 0001001, pp.1-76, ISSN 1102-1071.

Lackey, E., Vaughan, J. G. \& Frazier M. (2003), Experimental examination of static loading of self-tapping screws used for mechanical attachments to composite sandwich materials, Journal of Advanced Materials, 35(4), 48-56.

Mehaffey, J. \& Cuerrier, P. (1994), A model for prediciting heat transfer through gypsumboard/wood-stud wall exposed to fire, Fire and Materials, 18, 297-305.

NIST (1980), Thermal Properties taken from NIST website. http://fire.nist.gov/brflpubs, Original Reference: Report 7040. 384C Statens Provingaanstalt, Stockholm.

PateCornell, M. E. (1996), Uncertainties in risk analysis: Six levels of treatment, Reliability Engineering $\mathcal{G}$ System Safety, 54(2-3), 95-111.

Rubinstein, R. Y. \& Kroese, D. P. (2008), Simulation and the Monte Carlo Method, Second Edition, John Wiley \& Sons, Hoboken.

Sakji, S. (2006), Probabilistic modeling and experimental validation of heat transfer and thermomechanical analysis with damage of a cardboard-plaster-cardboard multilayer submitted to fire 
loads. PhD Thesis, Universit de Marne-la-Valle, France, 2006.

Sakji, S., Soize, C. \& Heck, J.-V. (2008), Probabilistic uncertainty modeling for thermomechanical analysis of plasterboard submitted to fire load, Journal of Structural Engineering (ASCE), 134(10), 1611-1618.

Sakji, S., Soize, C. \& Heck, J.-V. (2009), Computational stochastic heat transfer with model uncertainties in a plasterboard submitted to fire load and experimental validation, Fire and Materials, 33(3), 109-127.

Schueller, G. I. (2005a), Uncertainties in Structural Mechanics and Analysis-Computational Methods, Computer and Structures, 83(14), 1031-1150.

Schueller, G. I. (2005b), Computational methods in stochastic mechanics and reliability analysis, Computer Methods in Applied Mechanics and Engineering, 194(12-16), 1251-1795.

Serfling, R. J. (1980), Approximation theorems of mathematical statistics, John Wiley \& Sons, Hoboken, New Jersey.

Shannon, C. E. (1948) A mathematical theory of communication, Bell System Technology Journal, 27(14), 379-423 \& 623-659.

Soize, C. (2000), A nonparametric model of random uncertainties on reduced matrix model in structural dynamics, Probabilistic Engineering Mechanics, 15(3),277-294.

Soize, C. (2010), Information theory for generation of accelerograms associated with SRS, Computer-Aided Civil and Infrastructure Engineering (CACAIE), 25(5), 334-347.

Soize, C. (2012), Stochastic Models of Uncertainties in Computational Mechanics, Lecture Notes in Mechanics Series, Engineering Mechanics Institute (EMI) of the American Society of Civil Engineers (ASCE), Reston, VA, USA.

Spall, J. C. (2003), Introduction to stochastic search and optimization, John Wiley \& Sons, Hoboken, New Jersey.

Sultan, M. A. (1996), A model for predicting heat transfer through noninsulated unloaded steelstud gypsum board wall assemblies exposed to fire, Fire Technology, 32(3), 239-259.

Thomas G. (2002), Thermal properties of gypsum plasterboard at high temperatues, Fire and Materials, 26, 37-45.

Walter, E. \& Pronzato, L. (1997), Identification of parametric models from experimental data, Springer-Verlag, Londres-United Kingdom.

Wang, Y. C. (2008), Special issue on fire resistance of steel and composite structures, Steel And Composite Structures, 8(3), 170-264. 